\title{
Relationship Between Heart Rate Recovery and Disease Severity in Chronic Obstructive Pulmonary Disease Patients
}

\author{
Dolores Blanco-Almazan ${ }^{1,2,3}$, Daniel Romero ${ }^{1}$, Willemijn Groenendaal ${ }^{4}$, Lien Lijnen ${ }^{5}$, \\ Christophe Smeets $^{6}$, David Ruttens ${ }^{6}$, Francky Catthoor ${ }^{7,8}$, Raimon Jané ${ }^{1,2,3}$ \\ ${ }^{1}$ Institute for Bioengineering of Catalonia (IBEC-BIST), Barcelona, Spain \\ ${ }^{2}$ Universitat Politècnica de Catalunya $\cdot$ BarcelonaTech, Barcelona, Spain \\ ${ }^{3}$ Biomedical Resarch Networking Center in Bioengineering, Biomaterials and Nanomedicine, \\ Barcelona, Spain \\ ${ }^{4}$ imec the Netherlands/Holst Centre, Eindhoven, the Netherlands \\ ${ }^{5}$ Hasselt University, Hasselt, Belgium ${ }^{6}$ Ziekenhuis Oost-Limburg, Genk, Belgium \\ ${ }^{7}$ imec, Heverlee, Belgium ${ }^{8}$ KU Leuven, Heverlee, Belgium
}

\begin{abstract}
Chronic obstructive pulmonary disease (COPD) patients exhibit impaired autonomic control which can be assessed by heart rate variability analysis. The study aims to evaluate the cardiac autonomic responses of COPD patients after completing a conventional six-minute walk test (6MWT). Fifty COPD patients were included in the study, for which an ECG signal (lead II) was acquired by a wearable device, before, during, and after the test. We used the heart rate (HR) time-series to assess the heart rate dynamic during recovery. The heart rate recovery (HRR) marker was evaluated every $5 \mathrm{~s}$ after the GMWT and showed different dynamic trends among severity groups. We compared the HRR among patient groups classified according to the GOLD standard. Significantly larger normalized HRR values ( $n H R R$ ) were found in mild COPD patients $\left(n=23, G O L D=\{1,2\} ; n H R R_{1}=14.8 \pm 7.5\right.$ $\left.\%, n H R R_{2}=18.6 \pm 8.1 \%\right)$ compared to those with more disease severity $\left(n=23, G O L D=\{3,4\} ; n H R R_{1}=9.3 \pm 5.8 \%\right.$, $p=0.002$; and $\left.n H R R_{2}=13.7 \pm 6.7 \%, p=0.041\right)$. The largest differences were observed around the first $30 \mathrm{~s}$ of the recovery phase ( $n H R R=10.8 \pm 6.6 \%$ vs. $n H R R=5.6 \pm 4 \%$ $p=0.001)$. Our results showed a slower recovery for the severest patients, suggesting that cardiac parameters like the ones we propose here, may provide valuable information for a better characterization of COPD severity.
\end{abstract}

\section{Introduction}

The complex clinical condition of chronic obstructive pulmonary disease (COPD) patients requires the assessment of different aspects of the disease. These aspects include the level of airflow limitation, the impact of symptoms on the patient's status, and the risk of exacerbations and rehospitalizations [1]. Accordingly, the 6-min walk test (6MWT) is commonly used to evaluate the exercise capacity of chronic respiratory patients [2]. In particular, the heart rate (HR) dynamic evaluated during the recovery of the test has been suggested as a predictor of worsening of respiratory diseases $[3,4]$.

Patients with COPD have exhibited impaired autonomic control as assessed by heart rate variability (HRV) analysis [5]. However, it is unknown whether dynamic markers from the heart rate may identify autonomic dysfunction linked with disease severity and respiratory function. Consequently, other markers beyond the standard pulmonary ones are needed to provide a more complete assessment of the COPD condition.

The main objective of the study was to evaluate the cardiac autonomic responses of COPD patients after completing a conventional 6MWT. Different cardiac autonomic responses among COPD severity groups could provide additional information for the assessment of these patients.

\section{Material and Methods}

\subsection{Six-minute walk test}

The study included fifty COPD patients recruited during their consultation or rehabilitation session at Ziekenhuis Oost-Limburg (Genk, Belgium). The protocol consisted of three consecutive phases, including a five-minute resting phase, a walking phase, and a five-minute recovery phase while the electrocardiogram (ECG) was acquired using a wearable device. During the walking phase, the patients performed a 6MWT by walking as far as possible along 


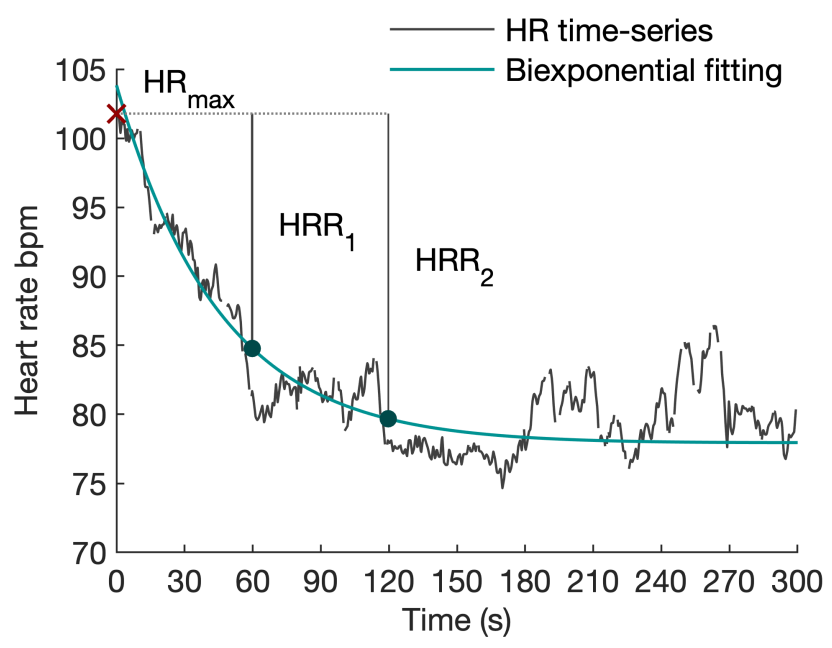

Figure 1. HR curve fitted using the biexponential approach described in [6]. $\mathrm{HRR}_{1}$ and $\mathrm{HRR}_{2}$ are computed as the decay from the $\mathrm{HR}_{\max }$ in the first and second minute.

a 45 meters corridor in 6 min [7]. This test is commonly used to assess the functional exercise capacity as a function of the walked distance [2]. On the other hand, the patients were sitting during the resting and recovery phases.

Demographic, anthropometric, and spirometry data were collected at the hospital. In particular, the spirometry data comprise the most common parameters used to assess COPD, the forced vital capacity (FVC), and the forced expiratory volume in one second $\left(\mathrm{FEV}_{1}\right)$.

The institutional medical ethics committee from Ziekenhuis Oost-Limburg approved the study (study ID 18/0047U). The patients provided written informed consent prior to study inclusion.

\subsection{ECG recording}

The measurements included the continuous ECG recording according to Lead II of Einthoven's Triangle. The acquisition was performed by a wearable research prototype device (imec the Netherlands, Eindhoven, the Netherlands) which allows the continuous recording of the raw ECG signal. The ECG signals were sampled at 512 $\mathrm{Hz}$.

All ECG signals were resampled to $500 \mathrm{~Hz}$ using spline interpolation. We obtained the inter-beat intervals by detecting the R-peaks using a wavelet-based method [8] followed by a visual inspection. The ectopic and abnormal beats were rejected to keep only the normal-to-normal $(\mathrm{NN})$ in the RR time-series.

\subsection{HRR markers}

We converted the RR time-series in beats/min to analyze the heart rate (HR) dynamic during the recovery phase af- ter the 6MWT. Then, the HR time-series were fitted using a biexponential approach presented in [6] that favors the calculation of the heart rate recovery (HRR) markers at specific time points. The HRR represents the decay in HR from the maximum HR $\left(\mathrm{HR}_{\max }\right)$ after the test. Our hypothesis is that such dynamic markers can help to assess COPD condition. Besides the standard timing for the HRR markers (first minute, $\mathrm{HRR}_{1}$, and second minute, $\mathrm{HRR}_{2}$ ), we alternatively computed the HRR every $5 \mathrm{~s}$ throughout recovery phase to analyze the HR dynamics in a higher time resolution. Furthermore, we normalized the HRR markers (nHRR) by the $\mathrm{HR}_{\max }$, as Fig. 1 shows.

\subsection{Statistical analysis}

The COPD patients were classified following the guidelines published in [1], resulting in four groups, GOLD 1, GOLD 2, GOLD 3, and GOLD 4, in order of severity.

We compared the HR dynamics among the four severity groups using the Kruskal-Wallis' test. The pairwise analysis included Bonferroni's p-value correction. The number of groups was reduced to two by joining the less severe groups, GOLD 1 and GOLD 2, and the severest groups, GOLD 3 and GOLD 4. We reduced the groups to have more balanced groups in terms of number of patients. We compared the HRR values for the resulting two groups.

\section{Results}

Fifty COPD patients were recruited in the study, 38 males and 12 females. Four patients were excluded from the analysis because of the presence of a pacemaker interfering the ECG signal (1) and a low signal-to-noise ratio (3). Therefore, the study population included forty-six patients whose demographic and anthropometric data are shown in Table 1.

Table 1. Demographic and anthropometric data for the study population

\begin{tabular}{lc}
\hline \hline & $\mathrm{n}=46$ \\
\hline Male (Female) & $34(12)$ \\
Age yr & $65.00(60.00-69.00)$ \\
Height cm & $169.50(164.00-178.00)$ \\
BMI kg/m & $24.85(22.27-29.04)$ \\
FVC \% pred & $86.45(72.20-110.90)$ \\
FEV $1 \%$ pred & $52.15(42.80-68.60)$ \\
GOLD 1 & $5(10.87 \%)$ \\
GOLD 2 & $18(39.13 \%)$ \\
GOLD 3 & $11(23.91 \%)$ \\
GOLD 4 & $12(26.09 \%)$ \\
\hline \hline
\end{tabular}

The data are presented as median (first - third quartile), and as the number (percentage) of patients for the grouping information. BMI: body mass index; FVC: forced vital capacity; $\mathrm{FEV}_{1}$ : forced expiratory volume in one second. 
Table 2. $\mathrm{HR}_{\max }$ and HRR of the COPD patients after the 6MWT

\begin{tabular}{|c|c|c|c|c|}
\hline & $\begin{array}{c}\text { GOLD } 1 \\
\mathrm{n}=5\end{array}$ & $\begin{array}{c}\text { GOLD } 2 \\
\mathrm{n}=18\end{array}$ & $\begin{array}{c}\text { GOLD } 3 \\
\mathrm{n}=11\end{array}$ & $\begin{array}{c}\text { GOLD } 4 \\
\mathrm{n}=12\end{array}$ \\
\hline $\mathrm{HR}_{\max }$ bpm & $90.67(71.10-108.67)$ & $100.93(86.47-113.34)$ & $113.09(103.77-126.58)$ & $107.18(93.92-119.06)$ \\
\hline $\mathrm{HRR}_{1}$ bpm & $11.44(6.68-14.43)$ & $12.65(9.64-19.44)$ & $10.11(8.24-12.37)$ & $10.08(3.88-12.99)$ \\
\hline $\mathrm{HRR}_{2} \mathrm{bpm}$ & $13.74(6.93-24.57)$ & $17.49(11.51-23.71)$ & $13.64(12.03-18.48)$ & $14.17(7.40-19.25)$ \\
\hline & \multicolumn{2}{|c|}{$\begin{array}{c}\text { GOLD } 1 \& \text { GOLD } 2 \\
\mathrm{n}=23\end{array}$} & \multicolumn{2}{|c|}{$\begin{array}{c}\text { GOLD } 3 \text { \& GOLD } 4 \\
\mathrm{n}=23\end{array}$} \\
\hline $\mathrm{HR}_{\max } \mathrm{bpm}$ & \multicolumn{2}{|c|}{$100.07(86.36-113.16)^{*}$} & \multicolumn{2}{|c|}{$110.11(98.04-124.99)^{*}$} \\
\hline $\mathrm{HRR}_{1}$ bpm & \multicolumn{2}{|c|}{$11.44(9.29-19.10)$} & \multicolumn{2}{|c|}{$10.11(5.99-12.37)$} \\
\hline $\mathrm{HRR}_{2} \mathrm{bpm}$ & \multicolumn{2}{|c|}{$16.81(10.76-23.70)$} & \multicolumn{2}{|c|}{$13.64(11.39-18.68)$} \\
\hline
\end{tabular}

The data are presented as median (first - third quartile) values. The severity classification followed the GOLD guidelines [1]. The * symbol denotes significant differences between groups p-value $<0.05$.
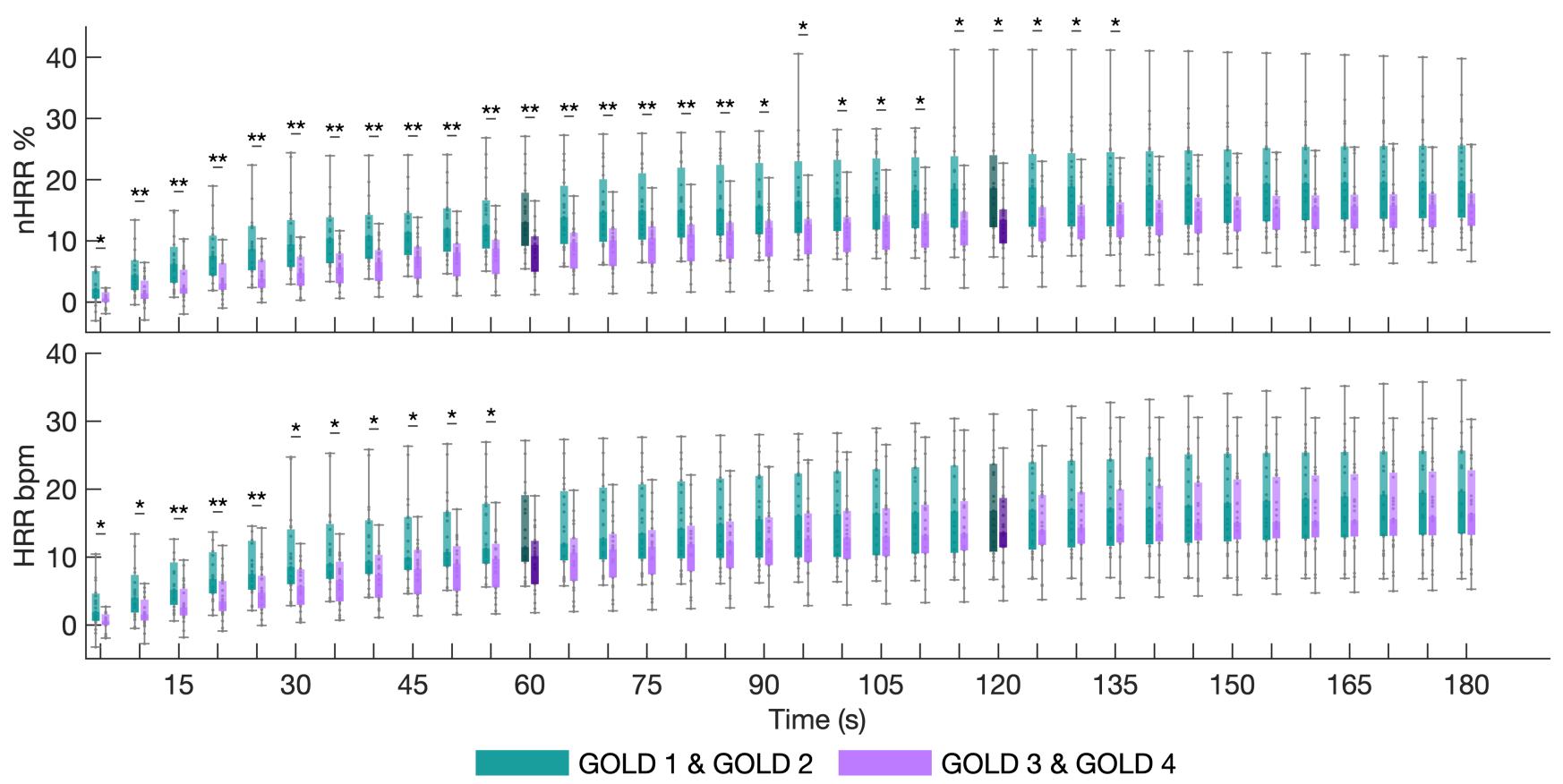

Figure 2. Evolution of the heart rate recovery, normalized by the $\mathrm{HR}_{\max }$ at the end of the test (nHRR) and the absolute value (HRR). The highlighted boxes represent the HRR in the first and second minutes. The significant differences were analyzed by the Kruskal-Wallis test with Bonferroni's correction in the pairwise p-values. ${ }^{*}$ denotes p-value $<0.05$ and $* *$ denotes p-value $<0.01$.

We evaluated the HR dynamics of the recovery period after the patients performed the 6MWT. We compared all the HRR values between the severity groups. Table 2 shows the $\mathrm{HR}_{\text {max }}, \mathrm{HRR}_{1}$, and $\mathrm{HRR}_{2}$ for two different analysis, one evaluated four severity groups, and the other two pooled groups. We found significant differences in $\mathrm{HR}_{\max }$ when the patients were classified into two groups, showing higher $\mathrm{HR}_{\max }$ for the severest patients. Regarding the HRR markers, we observed a tendency towards lower HRR values in the patients with worse COPD condition.

On the other hand, Fig. 2 shows both the absolute HRR values (upper panel) and the corresponding normalized values, nHRR (lower panel), measured every $5 \mathrm{~s}$. This figure shows the results for patients pooled in two severity groups. We observed different dynamic trends among the severity groups. Firstly, the nHRR showed significant differences until the first $135 \mathrm{~s}$ of the recovery period. In particular, the values were $\mathrm{nHRR}_{1}=14.8 \pm 7.5 \%$ and $\mathrm{nHRR}_{2}$ $=18.6 \pm 8.1 \%$ for the group GOLD $=\{1,2\}$ which were significantly higher compared to those with more disease severity GOLD $=\{3,4\}$, with $\mathrm{nHRR}_{1}=9.3 \pm 5.8 \%$ and $\mathrm{nHRR}_{2}=13.7 \pm 6.7 \%$. Note that the largest differences were observed around the first $30 \mathrm{~s}$ of the recovery phase, 
nHRR $=10.8 \pm 6.6 \%$ vs. $n H R R=5.6 \pm 4 \%$. Moreover, the absolute values, HRR, showed significant differences in the first $50 \mathrm{~s}$ as well. Overall, the results exhibit a slower recovery for the patients who had worse diagnoses (GOLD 3 and GOLD 4).

\section{Discussion}

The main objective of the present study was to investigate the cardiac autonomic responses of COPD patients after completing a standard 6MWT. We evaluated the HR dynamics during recovery from ECG signals acquired by a wearable device in forty-six COPD patients. The results showed that the most severe patients, GOLD 3 and GOLD 4 , had a slower recovery than the moderate patients.

The HRR measured in the first minute, $\mathrm{HRR}_{1}$, after the 6MWT has been suggested as a predictor of clinical worsening and mortality in respiratory patients suffering from idiopathic pulmonary fibrosis or pulmonary arterial hypertension $[3,4]$. These previous studies only evaluated the HRR markers at standard timing i.e., after the first and second minute. However, fitting the HRR curve allows us to increase the temporal resolution and evaluate these markers beyond the classical time points. This approach was presented in [6] to investigate the HRR time-course in Brugada syndrome patients during a submaximal exercise test. Although the study population and the test are quite different, our results show that this conceptual approach is suitable in the COPD population as well.

The presented results showed different dynamics in HR recovery among the COPD severity groups, particularly a slower recovery in the severest patients. We hypothesize that this slower recovery is related to a depressed response of the vagal stimuli reported in COPD patients by HRV analysis [5]. These differences were more significant at the beginning of the recovery, probably because the nature of the test in which is submaximal and the $\mathrm{HR}_{\max }$ did not increase excessively (see Table 2). Consequently, the HRR evaluated beyond the first and second minute provides valuable information about COPD severity and its cardiac consequences.

This study followed the GOLD guidelines for COPD assessment that include the evaluation of the airflow limitation and the symptoms of the patients [1]. In a preliminary phase of this study, we performed the same analysis but classifying the patients only by $\mathrm{FEV}_{1} \%$ pred. Using this classification, we did not find significant differences, and thus, these results are not presented. Accordingly, we can claim that nHRR and HRR markers provide more information about COPD conditions, not only related to airflow limitation. However, future studies should consider the implications of taking medications like betablockers in the HRR dynamics and markers.

We observed a slower recovery for patients who had worse diagnoses, probably due to a debilitated response of vagal control reactivation. The presented results suggest that, besides respiratory markers, cardiac parameters may provide valuable information for a better characterization of disease severity in COPD patients.

\section{Acknowledgments}

This project has received funding from the European Union's Horizon 2020 Research and Innovation Programme under the Marie Sklodowska-Curie grant agreement No. 846636. This work was also supported in part by the Universities and Research Secretariat from the Generalitat de Catalunya under Grant GRC 2017 SGR 01770 and, Grant FI-DGR, in part by the Agencia Estatal de Investigación from the Spanish Ministry of Science, Innovation and Universities and the European Regional Development Fund, under the Grant RTI2018 098472-BI00, and in part by the CERCA Programme/Generalitat de Catalunya.

\section{References}

[1] Global Initiative for Chronic Obstructive Lung Disease, "2020 Global strategy for diagnosis, management, and prevention of COPD," 2020.

[2] A. E. Holland et al., "An official European Respiratory Society/American Thoracic Society technical standard: field walking tests in chronic respiratory disease," European Respiratory Journal, vol. 44, no. 6, pp. 1428 LP - 1446, dec 2014.

[3] J. J. Swigris et al., "Heart rate recovery after 6-min walk test predicts survival in patients with idiopathic pulmonary fibrosis," Chest, 2009.

[4] O. A. Minai et al., "Heart rate recovery predicts clinical worsening in patients with pulmonary arterial hypertension," American Journal of Respiratory and Critical Care Medicine, 2012.

[5] M. Volterrani et al., "Decreased heart rate variability in patients with chronic obstructive pulmonary disease," Chest, vol. 106, no. 5, pp. 1432 - 1437, 1994.

[6] D. Romero et al., "A biexponential approach for assessing parasympathetic reactivation after submaximal exercise," in 2017 Computing in Cardiology (CinC), Sep. 2017, pp. 1-4.

[7] "ATS statement: Guidelines for the six-minute walk test," American Journal of Respiratory and Critical Care Medicine, vol. 166, no. 1, pp. 111-117, 2002, pMID: 12091180.

[8] J. P. Martinez et al., "A wavelet-based ecg delineator: evaluation on standard databases," IEEE Transactions on Biomedical Engineering, vol. 51, no. 4, pp. 570-581, April 2004.

Address for correspondence:

Dolores Blanco-Almazán

IBEC - Campus Diagonal-Besòs (UPC-EEBE)

Av. d'Eduard Maristany 16, 08019 Barcelona

dblanco@ibecbarcelona.eu 